\title{
An Insight into the Battery Degradation for a Proposal of a Battery Friendly Charging Technique
}

\section{Bikash Sah}

IIT Guwahati

Praveen Kumar ( $\sim$ praveen_kumar@iitg.ac.in )

IIT Guwahati

\section{Research Article}

Keywords: Battery, Battery Degradation, Charging Techniques, Electrochemistry, Fast Charging

Posted Date: August 9th, 2021

DOI: https://doi.org/10.21203/rs.3.rs-710085/v2

License: (9) This work is licensed under a Creative Commons Attribution 4.0 International License. Read Full License 


\title{
An Insight into the Battery Degradation for a Proposal of a Battery Friendly Charging Technique
}

\author{
Bikash Sah, ${ }^{1}$ Praveen Kumar, ${ }^{1 *}$ \\ ${ }^{1}$ Department of Electronics and Electrical Engineering, Indian Institute of Technology Guwahati, \\ Guwahati, Assam, 781039, India
}

*To whom correspondence should be addressed; E-mail: praveen_kumar@iitg.ac.in

Li-ion batteries are widely used in electric vehicles because of their promising characteristics that meet high specific power and energy density requirements. The only setback is the capacity fading due to degradation in Li-ion batteries. The rate of capacity fade in Li-ion batteries in EVs vary based on the charging rate, changes in internal cell temperature and external ambient temperature, and user driving patterns. Since the Li-ion battery is electrochemical, determining the actual cause of degradation at a particular instant and constraining the rate is a big challenge. Further, the causes are related to parameters which are chemical, electrical and mechanical. In this work, the causes of degradation are studied by analysing the variation of parameters for multiple charge types and rates at different ambient temperatures. The analysis leads to developing a new universal charging scheme suitable to fast charge battery at different ambient temperatures appropriately and constrain battery degradation. 


\section{Introduction}

Lithium-ion batteries have shown promising characteristics to meet the requirements of both hybrid and battery electric vehicles. Lithium-ion batteries were first commercialised in 1991 by Sony corporation (1). Higher energy density, specific energy and power density of Li-ion batteries are the major reason for wide usage in EVs. The equivalent weight of the lithium metal compound $(160 \mathrm{~g} \mathrm{Li} / \mathrm{kWh})$ used in the battery's negative electrode is low compared to other chemistries of secondary batteries (2). The size of the positive ion of lithium $\left(0.74 \mathrm{~A}^{\circ}\right)$ is also very small, providing ease to diffusion and efficient intercalation and deintercalation of $\mathrm{Li}+$ ions in electrodes of battery. The lower weight of Li metal leads to a higher energy density and specific energy, while the size of its ion helps increase the power density of the batteries. To date, researchers have proposed a variety of Lithium-ion chemistries that have either been commercialised or still under research $(1,3)$. The goals of the ongoing research in batteries for application in EVs are to improve the safety, thermal stability, energy density, rate of charge acceptance, cycle durability and reduce the manufacturing and environmental cost $(4,5)$.

Although Li-based chemistry is commercially used in EVs, it has limitations that hinder the acceptance. The Li compounds and the materials required for manufacturing Li-ion batteries should have the least impurity. Further, metals like lithium, nickel, cobalt and manganese are also costly. Hence, the development and manufacturing processes of Li-ion batteries is high (6).

Capacity fade due to ageing and the change in its rate due to variation in ambient temperature are the major issues related to Li-ion batteries. Calendar and cyclic ageing are the two types of ageing characterised in batteries. Calendar ageing is linked to the storage conditions of batteries, state of charge (SoC), and the storage area's ambient temperature. A higher storage temperature and SoC instigates the secondary reactions (7). On the other end, cyclic ageing is characterised by the degradation in the battery due to the charge-discharge cycle. Structural 
change in electrodes, solid electrolyte layer formation, chemical decomposition, or dissolution is a few resultants of cyclic ageing (8). Capacity fade of Li-ion batteries is a widely researched topic in literature (9). The literature highlights that the magnitude and pattern of applied current density, ambient and internal temperature of battery, packaging and mechanical stress have a major role in deciding the rate of capacity fade (10). The discharge pattern of the batteries also impacts the capacity fade, but the pattern in an EV is based on the requirement of current to drive the motor in the powertrain. Hence, instead of studying capacity fade due to discharge pattern in an EV, the sizing of entities in a powertrain is widely studied.

The directions of research in capacity fade is diverse. Research in electrodes (material, structure, chemistry and binders), electrolytes (chemistry and additives), the structure of the battery, tabs, parameter estimation, electrochemical modelling, mechanical modelling, causes of capacity fade, electrical modelling and charging techniques are commonly found in the literature $(10,11)$. However, they have a common question to answer- how can the battery be charged at the fastest possible rate with minimum capacity fade? Fast charge and low capacity fade are opposing goals which is shown in Figure S.7. Figure S.7 shows the increase in the rate of capacity fade with an increase in the charging rate. An increase in the charging rate corresponds to a decrease in the time to charge.

Further, the battery is an electrochemical system. Hence it requires more profound insights into the chemical and mechanical changes occurring inside the battery to claim the causes of capacity fade. Chemical degradation and mechanical degradation are the two types of degradation reported in the literature.

The processes of chemical and mechanical degradation and their dependency on the charging techniques and the ambient temperature have been described in the literature. However, there is a lack of studies that provide a deeper insight by describing the variation of both types of parameters of battery with change in charging technique. The parameters widely studied in 
the literature are inactive material, SEI layer thickness, overpotential, lithiation, porosity, tortuosity, and particle crack length $(10,12-14)$. The variation of the parameters results in the change in charging time, capacity, energy and power $(13,15)$. Moreover, the parameters are dependant on the ambient temperature of the battery. The studies related to the impact on the battery parameters with change in the ambient temperature with change in charging types are also missing in the literature. Hence, via this work, the authors describe the impact of $C_{\text {rate }}$ on the rate of chemical and mechanical degradation, the suitability of charging techniques at different ambient temperatures, and the suitability of the type of charging for high power and low power application. Further, a new charging technique suitable for all applications and ambient temperatures is proposed to fast charge with constrained degradation.

\section{Results}

The parameters of Li-ion batteries studied in the literature are either electrical or electrochemical, or mechanical in nature. The electrical parameters studied are impedance, SoC, SoH, capacity (fade, retention, relative, incremental, utilisation), energy efficiency, variations in OCV voltage, and specific energy are. Electrochemical parameters include side reactions rate, overpotential, active material volume concentration in electrodes, SEI (thickness, density, film resistance, potential), lithium loss, side reaction exchange current density, electrode potentials, and polarisation voltage. Porosity, electrode particle cracking, structural disordering, stress and expansion of cell (width and length) are the mechanical parameters studied in the literature. The rise in temperature, change in charging time, and rate of capacity fade results from variation in these parameters.

The $\mathrm{CC}-\mathrm{CV}$ is the oldest and standard technique described in the literature. Hence, all the new proposed charging algorithms have compared the variation of parameters listed above with CC-CV. Moreover, impedance is the most commonly studied parameter in earlier studies 
because of the possibility to relate with the variation of SoC and battery degradation $(14,16-$ 19). Table S.3 list the types of parameter studied for different types of charging techniques. Maximum parameters are studied in $\mathrm{CC}-\mathrm{CV}$ charging technique being the oldest and a standard charging technique. In contrast, the least number of parameters are studied in the temperaturebased charging technique (20).

The study of variation in the type of parameters for each charging technique is associated with the rate of battery degradation. Although the literature has described multiple studies on each charging technique to prove the suitability of fast charge and reduced battery degradation, there are lack of studies that associate all the three types of parameters -electrical, chemical, and mechanical. Further, only a few works describe the effect on the parameters due to an increase or decrease in the ambient temperature. Hence, the commercially used charging techniques$\mathrm{CC}, \mathrm{CCCV}$, pulse charging (with negative pulse and without negative pulse) and variable frequency/duty charge pulse will be analysed in detail to understand and associate the change in the three types of parameter with degradation phenomenon.

In this work, overpotential, the extent of lithiation in electrodes, inactive material volume fraction, SEI layer thickness in electrodes are studied as chemical parameters. The porosity, tortuosity, and the phenomenon of particle cracking in the electrodes are included in mechanical parameters, while the resultant parameters such as energy, power and capacity fade as analysed as electrical properties. Li-ion batteries undergo two reactions during charging and dischargingprimary intercalation reactions and secondary electrochemical reactions (also called as side reaction). The intercalation reactions are responsible for the charge and discharge of batteries. In contrast, the side reactions lead to the loss of lithium and materials, which intervenes in intercalation reactions called active materials. It also leads to electrolyte oxidation and reduction, passivation, structural disordering, particle cracking and thickening of the SEI layer. The resultants of side reactions lead to the capacity fade in the battery, which will be described in the 


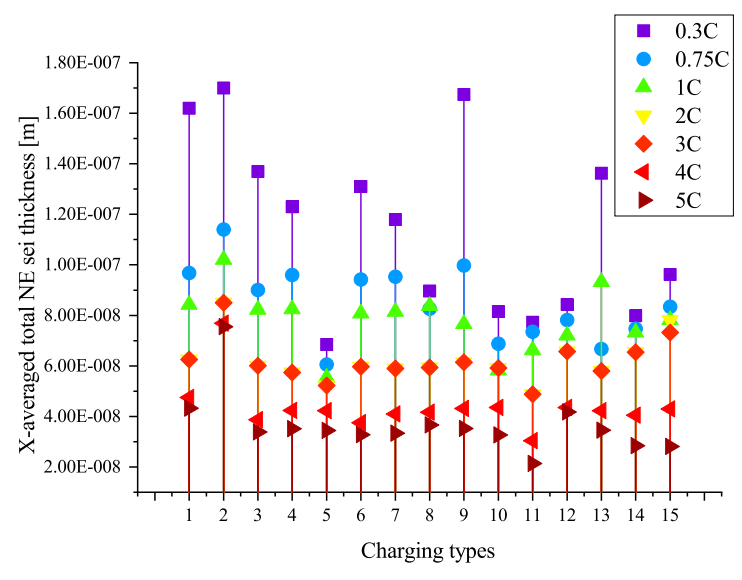

(a)

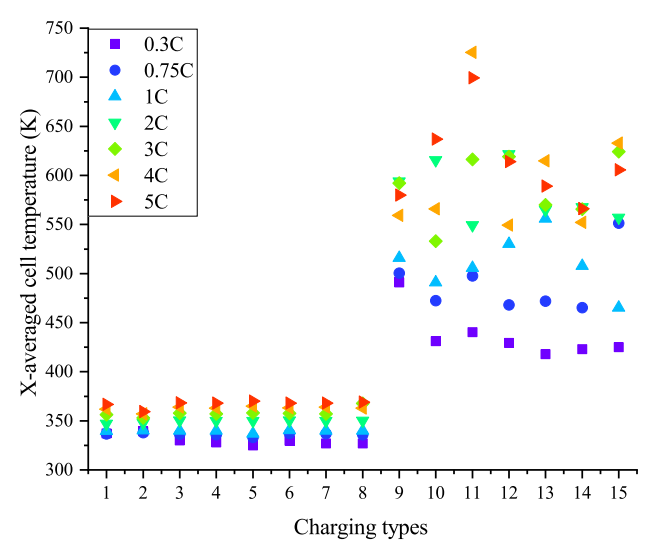

(c)

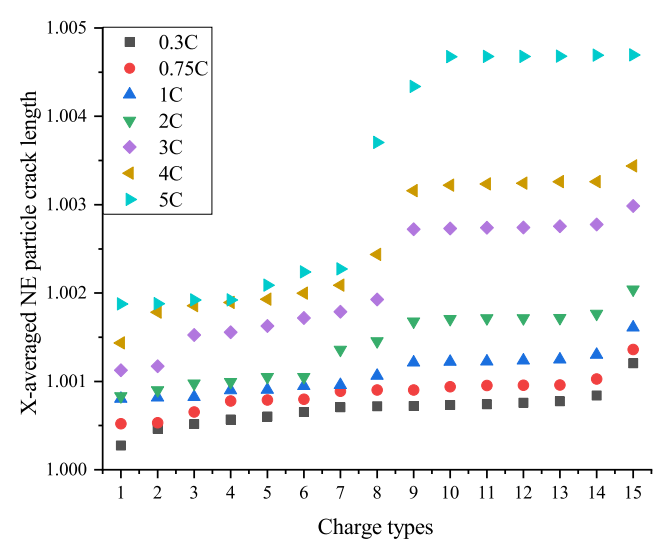

(b)

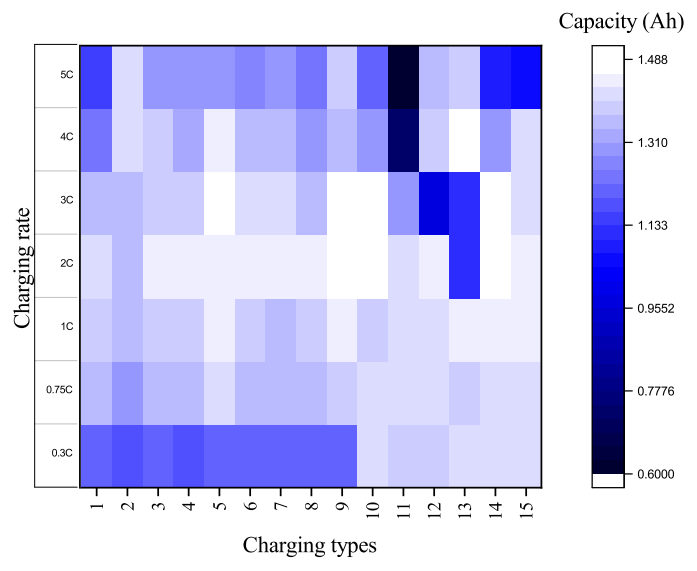

(d)

Figure 1: Variation of parameters of the batteries at different charging techniques and charging rates : (a) The X-averaged total negative electrode SEI thickness [m] decreases with increase in $C_{\text {rate }}$ as the chemical degradation is dominant at slower $C_{\text {rate }}$; (b) $\mathrm{X}$-averaged negative electrode particle crack length increases with increase in $C_{\text {rate }}$ because of the increased stress in electrode particles; (c) X-averaged cell temperature [K] is higher for higher $C_{\text {rate }}$ due to the increase in chemical kinetics. The discharge pulses further instigates the phenomenon because of the change in the direction of motion of ions and masses;(d) Capacity is a function of chemical and mechanical parameters and internal change in temperature. 
subsequent subsections. SEI layer thickness, particle cracking, change in internal cell temperature are the major parameters impacting the discharge capacity of batteries. Hence, they are included in the main study while others are described in the supplementary section.

Table 1: Summary of the variation of parameters with increase in the charging rate in different charging techniques. ${ }^{\dagger}, \#, \Downarrow, \Uparrow$, and * resembles decreasing, increasing, decreasing trend, increasing trend and negligible change, respectively.

\begin{tabular}{|c|c|c|c|c|c|c|c|c|c|c|c|c|}
\hline \multirow{3}{*}{ Sl. No } & \multirow{3}{*}{ Parameters } & \multirow{3}{*}{$\frac{\mathbf{C C}}{C_{\text {rate }}{ }^{\#}}$} & \multirow{3}{*}{$\frac{\mathbf{C C C V}}{C_{\text {rate }}^{\#}}$} & \multicolumn{4}{|c|}{ Pulse charging without discharge } & \multicolumn{5}{|c|}{ Pulse charging with discharge } \\
\hline & & & & \multirow{2}{*}{$t_{o n}^{\dagger}$} & \multirow{2}{*}{$t_{\text {on }}^{\dagger}$} & \multirow{2}{*}{$t_{o n} \& t_{o f f}^{\dagger}$} & \multirow{2}{*}{$C_{\text {rate }}{ }^{\#}$} & \multirow{2}{*}{$t_{o n}$} & \multicolumn{3}{|c|}{ Amplitude of discharge current } & \multirow{2}{*}{$C_{\text {rate }} \#$} \\
\hline & & & & & & & & & 0.5 & 1 & 2 & \\
\hline 1 & $\begin{array}{lr}\text { X-averaged } & \text { negative } \\
\text { electrode } & \text { inactive } \\
\text { material } & \text { volume } \\
\text { fraction } & \\
\end{array}$ & $\Downarrow$ & $\Downarrow$ & $\Downarrow$ & $\Downarrow$ & $\Downarrow$ & $\Downarrow$ & $\Downarrow$ & $\Downarrow$ & $\Uparrow$ & $\Downarrow$ & $\Downarrow$ \\
\hline 2 & $\begin{array}{lr}\text { X-averaged } & \text { total } \\
\text { negative } & \text { electrode } \\
\text { SEI thickness }[\mathrm{m}]\end{array}$ & $\Downarrow$ & $\Downarrow$ & $\Downarrow$ & $\Downarrow$ & $\Downarrow$ & $\Downarrow$ & $\Downarrow$ & $\Downarrow$ & $\Uparrow$ & $\Downarrow$ & $\Downarrow$ \\
\hline 3 & $\begin{array}{l}\text { X-averaged negative } \\
\text { electrode porosity }\end{array}$ & $\Downarrow$ & $\Downarrow$ & $\Downarrow$ & $\Downarrow$ & $\Downarrow$ & $\Downarrow$ & $\Downarrow$ & $\Downarrow$ & $\Uparrow$ & $\Downarrow$ & $\Downarrow$ \\
\hline 4 & $\begin{array}{l}\text { X-averaged negative } \\
\text { electrode tortuosity }\end{array}$ & $\Uparrow$ & $\Uparrow$ & $\Uparrow$ & $\Uparrow$ & $\Uparrow$ & $\Uparrow$ & $\Uparrow$ & $\Uparrow$ & $\Downarrow$ & $\Uparrow$ & $\Uparrow$ \\
\hline 5 & $\begin{array}{ll}\text { X-averaged } & \text { negative } \\
\text { electrode } & \text { reaction } \\
\text { overpotential }[\mathrm{V}]\end{array}$ & $\Uparrow$ & 介 & $\Downarrow$ & $\Downarrow$ & $\Downarrow$ & $\Uparrow$ & $\Downarrow$ & $\Uparrow$ & $\Downarrow$ & $\Uparrow$ & $\Uparrow$ \\
\hline 6 & $\begin{array}{l}\text { X-averaged negative } \\
\text { electrode extent of } \\
\text { lithiation }\end{array}$ & $\Downarrow$ & $\Downarrow$ & $\Downarrow$ & $\Downarrow^{*}$ & $\Downarrow$ & $\Downarrow$ & $\Downarrow$ & $\Downarrow$ & $\Uparrow$ & $\Uparrow$ & $\Downarrow$ \\
\hline 7 & $\begin{array}{l}\mathrm{X} \text {-averaged cell tem- } \\
\text { perature }[\mathrm{K}]\end{array}$ & $\Uparrow$ & $\Uparrow$ & $\Uparrow$ & * & $\Downarrow$ & $\Uparrow$ & $\begin{array}{l}\text { Low } \\
C_{\text {rate }}=\Uparrow \\
\text { and } \\
\text { High } \\
C_{\text {rate }}=\Downarrow\end{array}$ & $\Downarrow$ & $\begin{array}{l}\text { Low } \\
C_{\text {rate }}=\Uparrow \\
\text { and } \\
\text { High } \\
C_{\text {rate }}=\Downarrow\end{array}$ & $\Downarrow$ & $\Uparrow$ \\
\hline 8 & Capacity fade & $\Uparrow$ & $\Uparrow$ & $\Downarrow$ & $\Downarrow^{*}$ & $\Downarrow$ & $\Uparrow$ & $\Uparrow$ & $\Downarrow$ & $\Uparrow$ & $\Uparrow$ & $\Uparrow$ \\
\hline 9 & $\begin{array}{lr}\text { X-averaged } & \text { negative } \\
\text { electrode } & \text { particle } \\
\text { crack length } & \end{array}$ & $\Uparrow$ & $\Uparrow$ & $\Uparrow$ & $\Uparrow$ & $\Uparrow$ & $\Uparrow$ & $\Uparrow$ & $\Downarrow$ & $\Uparrow$ & $\Uparrow$ & $\Uparrow$ \\
\hline
\end{tabular}

\section{SEI layer thickness}

Side reactions in Li-ion batteries occur in three major regions viz. electrode-electrolyte interfaces, electrode-collector interface and electrolyte (21). Change in the equilibrium potential of the reactions during charging and discharging leads to instability in the electrolyte. The instability is accompanied by the start of side reactions within battery (22). The change in the equilibrium potential depends on the amplitude of the charging current and the types of charging. Hence, the concentration of the inactive materials and electrode-electrolyte interfaces or 
SEI layer thickness varies for different charging types and rates. Although the SEI layer is a by-product of side reactions, it is a required protective layer in graphite particles of negative electrodes in the battery. The lithium potential makes the electrolyte unstable and vulnerable to the reaction, which leads to the loss of lithium and a reduction in the overall capacity of batteries. Since it is a form of inactive material, the variation of the concentration of inactive material and the thickness of SEI layer are similar as shown in Fig. S.1 and Fig. 1a. The thickest SEI layer is formed in $\mathrm{CC}$ and $\mathrm{CCCV}$, followed by $\mathrm{CT}$, which is pulse charging with discharge. The SEI layer thickness for all charging rates is also highest in CCCV. During CCCV, the time for the $\mathrm{CV}$ phase is higher and increases with an increase in the number of cycles. During CV, the stress due to electrochemical reactions and temperature is lower than the CC phase, leading to stable SEI formation (8). Hence, with an increase in the number of cycles, the SEI layer keeps becoming thicker. Further, the formation of SEI is also higher at lower charging rates for the same reason as seen in Fig. 1a.

The plot in Fig. 1a shows that pulse charging has the potential to reduce the SEI layer thickness. The SEI layer thickness in charge type 3 to 8 , which is pulse charge without discharge, varies with the change in the $t_{o n}$. The thickness is highest in charge type 3, which has the maximum $t_{o n}$, while charge type 5 has the lowest because of the least $t_{o n}$. The $t_{o f f}$, too, impacts the thickness of the SEI layer. With a decrease in the $t_{\text {off }}$, the SEI layer thickness is reduced. A reduced $t_{o n}$ as well as the $t_{o f f}$ help to suppress the thickness of the SEI layer. A larger $t_{o n}$ at a lower $C_{\text {rate }}$ act similar to CCCV. Even at a higher $C_{\text {rate }}$, the rest phase of the pulse will help to stabilise the SEI layer leading to thickening with an increase in the number of cycles. Hence, a decrease in $t_{o n}$ and $t_{o f f}$ help reduce the SEI layer's thickening.

The variation in the change of SEI layer thickness for pulse charging with discharge is shown in charge type 9 to 15 in Fig. 1a. Majority of the pulse charging with discharge charge type help in reducing the SEI layer thickness. The SEI layer thickness has a reducing trend for charge 
type 9 to 11 , although the variation is not much for charge type 10 and 11 . The amplitude of discharge current, too, had a role in the change in the SEI layer thickness. When the amplitude of the discharge current is equal to the average current (charge type 9 to 11), with the reduction in $t_{o n}$, the SEI layer thickness is also reduced.

Further, when the amplitude of the discharge current is equal to half of the average current (charge type 12 and 13), a higher on-time resulted in less SEI layer thickness. On the contrary, when the amplitude of discharge current is equal to the twice average current, a higher on-time resulted in a thicker SEI layer. The change in the polarity of the charge pulses leads to a similar variation of equilibrium potential. When the amplitude of the current in the discharge pulse is more than the charge pulse, the equilibrium potential falls below the electrolyte's stability limits, accelerating the SEI formation. The rest phase further helps to stabilise the SEI formed, leading to a thick SEI layer in comparison when the amplitude of the current in the discharge pulse is less than or equal to the charge pulse. The charge type with amplitude of discharge current equal to the average current and least $t_{\text {on }}$ and rest time have thinnest SEI layer formed over the negative electrode.

\section{Particle crack length}

Particle cracking is a form of mechanical degradation in battery observed in the electrode particles. The stress in electrode particle are commonly modelled or experimentally reported due to intercalation/deintercalation reaction and changes in internal cell temperature variations or ambient temperature variations. The crack in the particles results in exposure to the surface of active materials leading to side reactions. These side reactions further lead to heat generations, amplifying the phenomenon of stress and side reactions due to an increase in cell kinetics (23). Hence, an increase in mechanical degradation increases the chemical degradation and viceversa. 
Fig. 1b shows the variation of particle crack length on the application of different types of charging techniques at different charging rates. The slower charging rates resulted in the least particle cracking. There is an increase in the particle crack length on going from left to right. The $\mathrm{CC}$ and $\mathrm{CCCV}$ resulted in the least crack length, although $\mathrm{CCCV}$ has higher values when compared to CC. Since at higher potential of battery, the stress on the particles of electrodes is higher, CCCV resulted in higher crack length. In CC, the battery is allowed to settle with a reducing charge current. Since the battery is not allowed to settle and overpotential remains higher, the stress due to increased potential of battery and saturation of electrodes is not experienced. While in the case of $\mathrm{CCCV}$, the battery keeps on charging after switching to $\mathrm{CV}$. With increase in potential, the stress on particles of electrodes continue to increase. Hence, the particle crack length is more than CC.

The pulse charging techniques that resulted in a better candidate for fast charge when previous parameters were considered do not perform well. The reason behind the increase in the crack is related to the heat generation during the intercalation and deintercalation reaction. The heat generation adds to the internal battery temperature rise, increased chemical kinetics, and stress in the electrodes particle. The higher the rise in internal cell temperature (Fig. 1c), the higher is the particle crack length. Hence, a change in the ambient temperature might result in an increase or decrease in particle cracking. During pulse charging with discharge, the increase in the amplitude of the discharge pulse resulted in a further increase in the particle crack length.

\section{Cell temperature}

The internal cell temperature is a major factor impacting the capacity fade of batteries. The temperature brings changes in equilibrium potential of reactions within the battery, the chemical kinetics which affects the rate of side reactions, SEI layer formation and erosion, the diffusivity of charge and mass in electrodes, stress in battery, structure disordering or electrodes and overall 
geometry, and safety in the operation of battery $(24,25)$.

A higher $C_{\text {rate }}$ resulted in an increase in the heat generation due to rapid diffusivity of charges and increased stress in the electrode particles. The conventional CC and CCCV had control on the rise in the internal cell temperature. However, during pulse charging with discharge, the temperature rose to a very high value. The temperature rise is related to the ease in the diffusivity of charge in the battery both during intercalation and intercalation. With an increase in the SEI layer thickness, decrease in porosity and increase in the tortuosity of electrodes, the charge and mass transfer are offered resistance. The increase in the resistance adds to the heat generation and impacts the stability of electrolyte.

The impact of the increase in internal cell temperature is deteriorating in nature. The increase in the temperature induces stress in the electrode particles and erodes the SEI layer. On erosion, the surface of active material or electrode particles are unlatched to further side reactions. Apart from forming the SEI layer, the side reactions leave behind residual inactive materials and gases, which are generated due to phase transition from solid to gas or liquid to gas. An uncontrolled rise in the temperature is imperative to lead to thermal runaway and harm the safety of the battery being charged pr discharged. Fig. 1c infers that conventional CC and CCCV and pulse charging without discharge are effective methods to perform fast charge with a limited rise in internal battery temperature.

\section{Discharge capacity}

Discharge capacity is the final parameter of the battery, which determines the performance of the battery. Discharge capacity depends on various parameters discussed in previous subsections. The reduction in capacity fade depends on the $C_{\text {rate }}$ and the types of charging techniques as shown in Fig. 1d. The $C_{\text {rate }}$ impacts the rate of chemical kinetics, equilibrium potential and stress due to intercalation reactions. An increase in the mentioned parameters increases the 
overpotential while vice-versa reduce the overpotential. At higher $C_{\text {rates }}$, the overpotential rises and leads to an increase in the terminal voltage. Since the battery terminal voltage reaches the cut-off potential much earlier, the electrode is not lithiated fully. The battery does not charge to the full range of SoC. When CCCV is used to charge, the CV part of charging allows the battery to be charged in a wider SoC range as the rise in overpotential is countered by a fall in current. Further, the time to charge also impacts the reduction in discharge capacity. A larger time period will lead to increased stress on electrode particles due to increased concentration gradients (26). Increased stress adds to SEI erosion and further side reactions leading to loss of active particles and Li-ions (27).

In Fig. 1d, it can be seen that CCCV leads to larger capacity loss at slower $C_{\text {rate }}$ when compared to $\mathrm{CC}$, but at a higher $C_{\text {rate }}$, the $\mathrm{CC}$ leads to larger capacity loss. A similar pattern is seen in the case of pulse charging without discharge also. The $t_{o n}$ and $t_{\text {off }}$ time also impacts the reduction in discharge capacity. A decrease in $t_{o n}$ and $t_{o f f}$ are instrumental in countering the reduction in discharge capacity. Contemplating, it is observed that higher charging rates are a possible solution with pulse charging without discharge. However, $2 \mathrm{C}$ is found to be an optimal Crate.

Looking into the pulse charging with discharge, an increased $t_{\text {on }}$ leads to a similar pattern of reduction in discharge capacity. The reduction in $t_{\text {on }}$ helps to reduce the rate of reduction of discharge capacity. The amplitude of discharge pulse during charging too impacted the change in discharge capacity. When the amplitude of discharge pulse is more than or equal to the average charging current, the discharge capacity reduces drastically. The drastic reduction can be related to the change in the equilibrium potential of reactions and the overpotential during charge, rest and discharge durations. The rise in internal cell temperature due to an increase in cell kinetics cannot be neglected while selecting an optimal charging technique to fast charge. The pulse charging with discharge increases the internal cell temperature to a higher value, 
leading to thermal runaway. Considering the reduction in discharge capacity, CT5 is the best technique to charge the battery at higher $C_{\text {rate }}$.

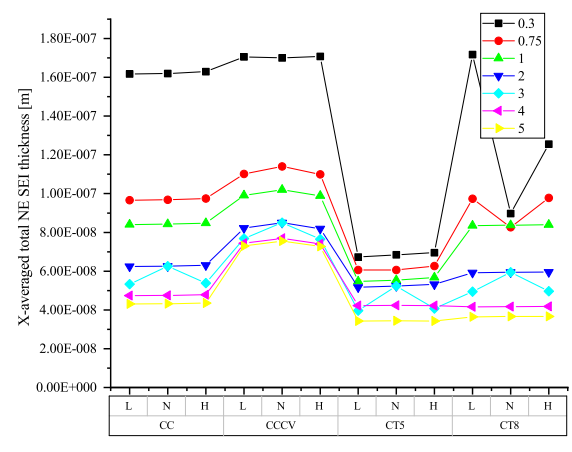

(a)

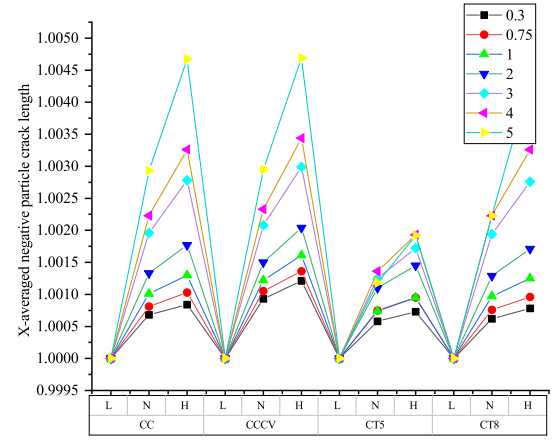

(b)

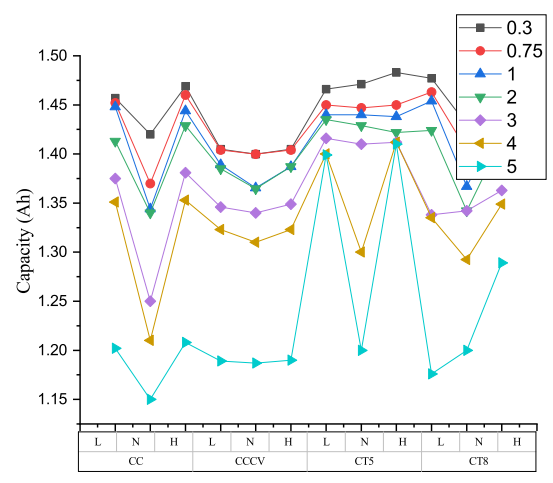

(c)

Figure 2: Variation of different parameters of the batteries: (a) An appropriate value of $t_{\text {on }}$ and $t_{\text {off }}$ help in reduction in the X-averaged negative electrode SEI thickness [m]; (b) The Xaveraged negative electrode particle crack length is also least when CT5 is used; (c) The variation of capacity fade is the most at higher $C_{\text {rate }}$, and CT5 is found to be helpful in constraining the rate of capacity fade.

\section{Comparison of the results with the change in ambient temperature}

Various works in literature have reported the impact of ambient temperature in operation and ageing mechanisms of the battery at different $C_{\text {rate }}$. However, the effect of the charging types is a not widely discussed topic in the literature, although a few works suggest charging techniques 
for extreme temperature conditions (28). Hence, this work is further extended to analyse the impact of the charging technique at two extreme temperatures - $318.15 \mathrm{~K}\left(45^{\circ} \mathrm{C}\right)$ and 273.5 $\mathrm{K}\left(0^{\circ} \mathrm{C}\right)$. The temperature changes the rate of formation of inactive materials, including SEI, erosion or decomposition of SEI and instability in electrolyte resulting in reduction reactions with active material. Rise of internal temperature above a certain level $\left(125^{\circ} \mathrm{C}\right.$ to $\left.180^{\circ} \mathrm{C}\right)$ can lead to thermal runaway, venting, and complete damage of battery (29).

The previous results, which were at $298.15 \mathrm{~K}\left(25^{\circ} \mathrm{C}\right)$, are compared with the results obtained at two extreme temperature for selected four types of charging techniques. CT5 and CT8 variants of pulse charge with discharge resulted in the best performing charging types based on the previous simulations at normal ambient temperature. Hence, to reduce the simulation time, further simulations are performed on only four types of charging techniques, viz. CC and CCCV being conventional, and CT5 and CT8 are only simulated at two extreme temperatures.

Fig. 2 and Fig. S.6 shows all the parameters studied to determine the performance of the battery at different charging rates and temperatures. Three different temperatures are represented by "L" $\left(273.5 \mathrm{~K}\left(0{ }^{\circ} \mathrm{C}\right)\right)$, "N" $\left(298.15 \mathrm{~K}\left(25^{\circ} \mathrm{C}\right)\right)$ and "H" $\left(318.15 \mathrm{~K}\left(45^{\circ} \mathrm{C}\right)\right)$. The parameters analysed are those presented in Fig. 1. The difference in the formation of inactive material for different $C_{\text {rate }}$ is the least in the case of CT5. For CC, at lower $C_{\text {rate }}$, the variation in the formation of inactive material is negligible with a change in the temperature. The change in overpotential of the battery is also less; thereby, the changes in SEI layer thickness, porosity, and tortuosity also follows a similar pattern. The parameter which shows differences are the lithiation, particle crack length and capacity. The changes in the lithiation is related to the rate of diffusivity, which changes with the change in the temperature. At lower temperature, higher resistance to diffusivity is found in the batteries, resulting in decreased lithiation. With an increase in the temperature, the Li-ions and mass transfer rate increases with an increase in chemical kinetics. As the lithiation increases, there is an increase in the stress in the particles of 
the electrode. Hence, as expected, the particle cracking increases with the temperature.

The changes in temperature does not results in a major change in the parameter when CCCV is used, although there is a rise in the formation of inactive materials, SEI layer thickness, overpotential, lithiation and tortuosity. Porosity decreases while particle crack length does not show any major difference with CC. CT5, which is found to be the best charging technique, shows the least variation in values of parameters at different charging rates. The decrease in the $t_{o n}$ and $t_{o f f}$ resulting in the changes as described in previous subsections. The results with reduced $t_{o n}$ and $t_{o f f}$ aligns with the finding in (30) where it is shown that a low-frequency diffusion leads to higher impedance of the battery. The impedance of the battery is due to an increase in the growth of the SEI layer and the formation of inactive materials. CT8, which follows the CT5 in performance, has a similar results pattern as found in CC, CCCV and CT5.

\section{Suitability of type of charging for high power or high energy applications}

The degradation of Li-ion batteries in EVs also depends on the application. The EVs in transport sector ranges from small two-wheeler to large bus application. The fast EV racing cars are not far behind in tracks. The variation in the requirements of torque and speed of EV motor changes the discharge pattern of batteries. Hence, the rate of degradation also varies for the same battery used in a different application. In this work, an analysis to determine the performance of battery for the higher energy or higher power applications is also done. Fig. 3 shows the plot of terminal power vs energy plots for different charging techniques and $C_{\text {rate }}$.

Fig. 3a shows the plot for lower ambient temperature. CT5 performs the best up to $2 C_{\text {rate }}$ for all the temperature conditions under study. The CCCV becomes a competitor at normal temperature conditions, but CT5 still outpaces. Further, CCCV is performs worst at low and high ambient temperature regions. The results clearly depict the requirements of different charging pattern for different types of vehicles. Fig. $3 b$ is the plot for normal ambient temperature range. 


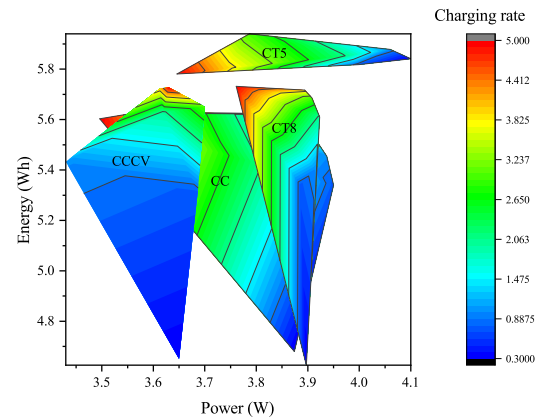

(a)

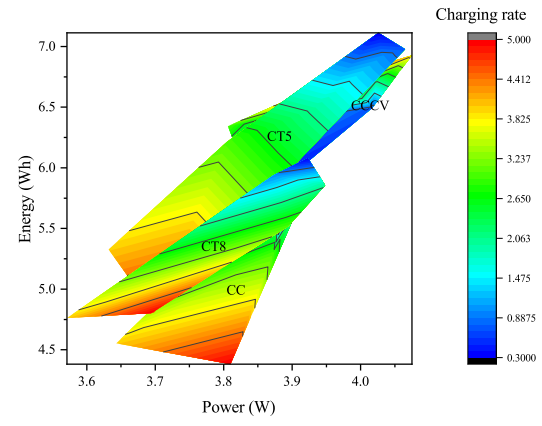

(b)

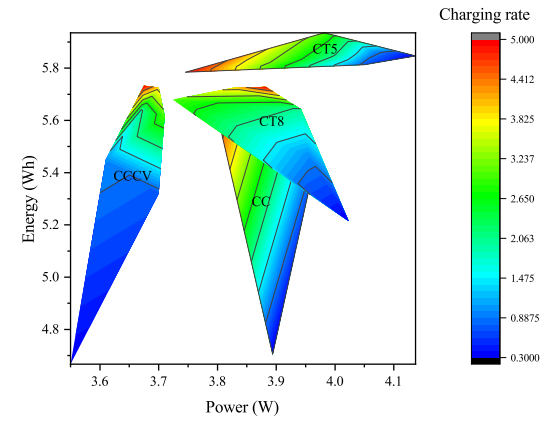

(c)

Figure 3: Variation of terminal power and energy of the battery for different charging techniques and charging rate at different temperatures (a) Energy vs power plot at very low ambient temperature; (b)Energy vs power plot at normal ambient temperature; (c)Energy vs power plot at high ambient temperature

CT5 and CCCV are close competitors while CT8 and CC are far behind and not suitable for use. From Fig. 3c, which is the plot for higher ambient temperature range, CT5 resulted in the best charging technique. The higher charging rate still remains a challenge for all the ambient temperature conditions. The solution to the problem is discussed in the next section.

\section{Discussion and outlook}

The benefits of Li-ion batteries and their wide application in EVs have developed research interest to meet the challenges. All the challenges in EVs converge to one- battery degradation $(4,8)$. The rate of battery degradation changes with the change in utilisation of EV battery. The utilisation depends on the EV driver behaviour, temperature of the environments and type of utilisation of EVs (high energy or high power applications). Higher energy and power density requirements result in a series-parallel connection of cells, which adds to the challenges of making the pack safe, durable, and lower cost. The battery degradation is triggered by the change in the equilibrium potential of the reactions in the battery. The thermodynamic force to drive the reactions in the battery is associated with equilibrium potential. Hence, changes in 
the internal battery or external ambient temperature lead to a variety of chemical and structural alternations (31).

An increase in the temperature leads to heighten kinetics of both intercalation-deintercalation and side reactions. The inactive material formed during elevated temperature has a different morphology in comparison with normal temperature. Most importantly, the SEI layer composition changes. Studies are performed using differential scanning calorimetry and accelerated rate calorimetry to determine the cell or electrode behaviour $(32,33)$. These studies disclosed the phenomenon of self-heating due to exothermic side reactions. The selection of electrolyte salt has a significant role in the temperature rise. The elevated temperature further erodes the existing SEI layer over the active material. The eroded SEI either dissolve or re-precipitate, leading to the restructuring of damaged SEI and more side reactions. More stable SEI and inorganic products are formed, such as lithium fluoride and lithium carbonate (34). Further, these stable products are less penetrable for Li-ions, thereby decreasing SEI's overall diffusivity and ionic conductivity.

At low temperatures, the degradation of the battery is not dominated due to changes in the SEI formation instead by the phenomenon of lithium plating and dendrite formation (13, 35). This study does not consider these two phenomena. Hence the related results are not presented. However, the literature states that, at low temperature, the equilibrium potential of intercalation reactions drops close to the lithium metal potential (36). Further, the viscosity changes in the electrolytes are observed, leading to a decrease in the diffusivity of li-ion into electrolytes and electrodes. Li-metal reactions in the electrolyte lead to side reactions, which accelerated the ageing process and increased capacity loss. The saturation of electrodes due to slow diffusion and Li's settlement around electrodes adds to the increase in local potential. Hence, the possibility of Li metal plating or dendrite formation increases.

The discussions in this subsection help gain insights into different types of charging tech- 
niques discussed in this work. The conventional techniques viz $\mathrm{CC}$ and $\mathrm{CCCV}$ have shown increased chemical degradation during lower $C_{\text {rate }}$ and increased mechanical degradation during higher $C_{\text {rate }}$. The inactive materials and SEI layer formed at all the $C_{\text {rate }}$ is higher when compared to pulse charging without discharge, as seen in Fig. S.6a and Fig. 2a. Similarly, the particle cracking length is also higher. The CT5 has shown the best results in terms of all the parameters analysed in Fig. 2. Even when the ambient temperature is taken into consideration, the performance does not deteriorate. Hence, pulse charging technique without discharge pulse with a reduced $t_{\text {on }}$ and $t_{\text {on }}$ can be a suitable option to go for fast charging and constrain the battery degradation. The value of $t_{o n}$ and $t_{o f f}$ is an optimization problem given in (37).

Although the CT5 results are optimal for all temperature conditions analysed in this work, the design of new charging techniques will be indispensable at extreme temperatures such as $-20{ }^{\circ} \mathrm{C}$ and $80^{\circ} \mathrm{C}$. Further, each charging technique has advantages and disadvantages that can be utilised to charge in extreme environmental conditions. The amalgamation of the charging techniques directs us towards the developing a new rule-based charging strategy for Li-ion batteries. Further, for high energy and high power applications as well, the rules can be framed by selecting the suitability of charging type from Fig. 3, monitoring the rise in internal battery temperature, and appropriately varying the $C_{\text {rate }}$.

The rule-based charging strategy should incorporate the battery electrochemistry, present battery health parameters, environmental conditions, user requirements and grid conditions. Hence, a new rule-based charging strategy is proposed to fast charge with reduced battery degradation in this work. Different types of electrochemistry of Li-ion battery are commercially available such as Lithium Cobalt Oxide (LCO), Lithium Manganese Oxide (LMO), Lithium Iron Phosphate (LFP), and Lithium Titanate (LTO). The battery electrochemistry is to be considered because of the variation in ability to fast charge, performance, lifespan, specific power, and energy. The present battery health conditions will help to determine the ageing of the bat- 
tery. Capacity estimated by the BMS or any types of the model or the previous battery charge and discharge profile data will help determine the battery's ageing. The environmental condition includes the temperature of the region. User requirements can be a fast, medium and slow charge. The grid conditions should also be incorporated to avoid the impact of uncoordinated charging, which leads to voltage imbalance and instability (38-40). The electric grid can be at peak load, off-peak load or normal condition.

Assume an EV arrives at a charging station with LTO battery electrochemistry. The battery is new and can charge to full capacity as communicated by the BMS. The temperature of the region of the charging station is below normal $\left(-10^{\circ} \mathrm{C}\right)$. The user opts to fast charge, and the grid is at peak load condition. For the given scenario, if a fast charge is performed, the grid will be overburdened. Hence, the user will be given an option to go with a slow or medium charge rate. If the user wants to continue the fast charge, the service will not be denied, and fast charging will be selected. The only condition to be evaluated is the ambient temperature. Since the temperature is very low, the battery is under stress, and the internal resistance is high. The rise of the internal resistance is due to the low ionic and mass diffusivity. Suppose the battery's internal temperature is increased, the viscosity of the electrolyte and the overall rate of diffusion can be brought to normal. The analysis in the previous subsection has shown that pulse charging with discharge can help in increasing battery's internal temperature. Hence, the battery undergoes a pulse charging with discharge for a period of time until the battery's internal temperature becomes normal. Later, with controlled battery temperature monitoring, fast charging can be performed using a charging pattern, leading to the least battery degradation. For instance, in this work, CT5 is found to be the best performer. Similarly, for LTO battery, an optimal charging pattern can be proposed.

The advantages of the rule-based charging system lie in the ease of implementation. The charging station developer can predefine a set of rules based on the changes in the climatic 
condition, nature of the user and their requirements of fast charge, condition of the electric grid over a period of time. The charger should be designed to meet the requirements of different charging strategies. A set of rules can be framed and fed to the controller of the charger. Every time a user connects for the charge, as per requirements, the rule will ensure the best services for both EV user and the electric grid. The assurance of the least battery degradation will advantage the EV user, and the electric grid will have the least impact of uncoordinated fast charge.

The disadvantages of such rule-based charging are data requirements related to battery parameters that need to be considered to fast charge with reduced battery degradation. Further, robust communication needs to be established with the grid for real-time monitoring of loading. The charger should also be capable of communicating with EV battery BMS. A computational signal processing board or connected infrastructure is also required to monitor and control the charging of EV batteries. These requirements make the charging systems costly, but an increase in cost will be compensated by avoiding ageing of battery and losses of power system operators due to uncoordinated charging. The benefits of Li-ion batteries and their wide application in EVs have developed research interest to meet the challenges. All the challenges in EVs converge to one- battery degradation Table 2 shows a rule base developed considering all the five parameters. 

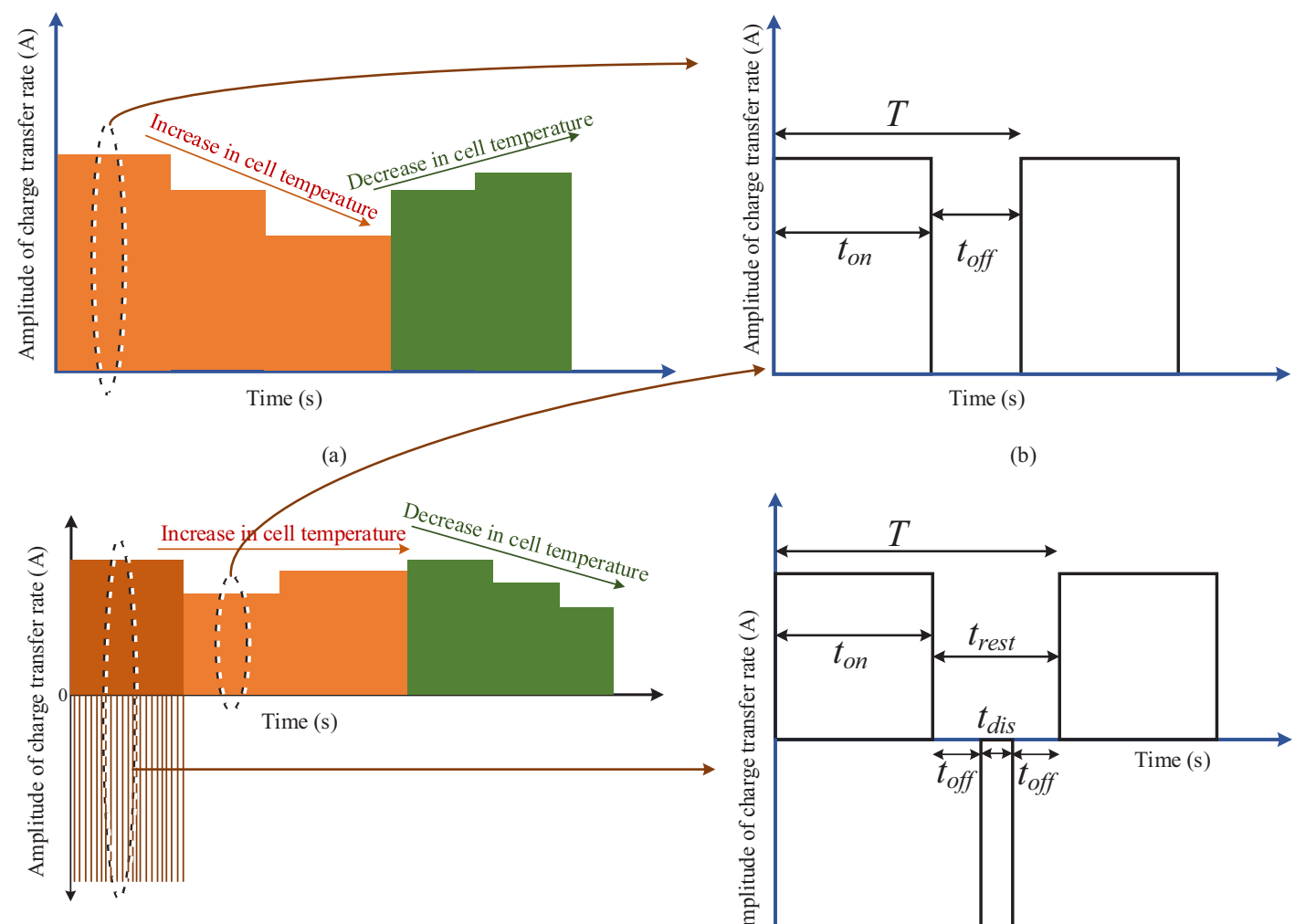

(b)

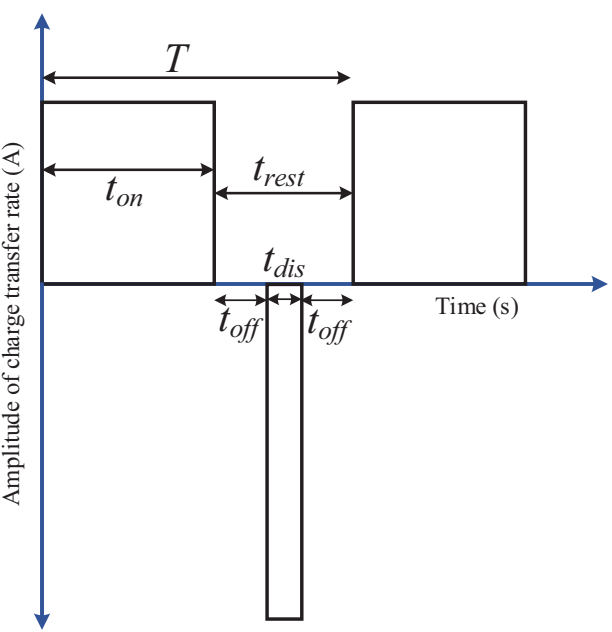

(c)

(d)

Figure 4: Proposed charging technique: (a) The charging pattern that is suitable for charging at normal or high ambient temperature. The increase and decrease in cell temperature is countered by reducing and increasing the amplitude of positive pulse current. (b) The pattern of pulse in which $T, t_{o n}$ and $t_{o f f}$ are required to be computed to constrain battery degradation. (c) The charging pattern that is suitable for charging at extreme low temperatures. The discharge pulse of more than average charging current help to increase internal cell temperature. The increase and decrease in this case is controlled by shifting from pulse charging with discharge to without discharge and increasing or decreasing the amplitude of charge current. 
Table 2: Example rule set for charging: The set of rules are designed based on the results obtained for the selected battery in this work. The user selection is not demonstrated in the process when the grid is in peak load hours. Further, frequency, duty cycle of the pulses and amplitude of charge and discharge pulse should be either computed online by preset optimisation algorithms or set as a predefined value determined based on experiments/simulations for any charge technique (CT5, CT9 or any pulse charging with discharge) defined in the table.

\begin{tabular}{|c|c|c|c|c|}
\hline Sl.No & $\begin{array}{l}\text { Present } \\
\text { battery } \\
\text { health }\end{array}$ & $\begin{array}{l}\text { Environmental } \\
\text { condition }\end{array}$ & $\begin{array}{l}\text { 1User re- } \\
\text { quirement }\end{array}$ & Charge technique \\
\hline 1 & New & Low & Fast & $\begin{array}{l}\text { Start charging by a high charge current pulse or pulse charging with discharge (similar } \\
\text { to CT9*). With an increase in the internal cell temperature, charge technique similar to } \\
\text { CT5* can be used to fully charge. }\end{array}$ \\
\hline 2 & New & Normal & Fast & Start charging with charging technique similar to CT5*. \\
\hline 3 & New & High & Fast & $\begin{array}{l}\text { Start charging by a technique similar to CT5* and monitor the internal temperature. With } \\
\text { the rise in the internal cell temperature, the } C_{\text {rate }} \text { should be reduced to constrain the rise. } \\
\text { On stabilising the rise in internal cell temperature, } C_{\text {rate }} \text { can be increased again. }\end{array}$ \\
\hline 4 & Half-life & Low & Fast & $\begin{array}{l}\text { Start charging by using a technique similar to CT9*, with amplitude of discharge pulse } \\
\text { lower than the average charge current. Monitor the rise in internal cell temperature. With } \\
\text { the rise in internal cell temperature, shift charging to CT5. The internal cell temperature } \\
\text { should be monitored to reduce } C_{\text {rate }} \text { on a rapid rise. }\end{array}$ \\
\hline 5 & Half-life & Normal & Fast & $\begin{array}{l}\text { Start charging using a technique similar to CT5 while monitoring the internal cell temper- } \\
\text { ature. An increase in internal cell temperature near stability limits should be constrained } \\
\text { by reducing } C_{\text {rate }} \text {. }\end{array}$ \\
\hline 6 & Half-life & High & Fast & $\begin{array}{l}\text { Start charging using a technique similar to CT5 and monitor the rise in internal tempera- } \\
\text { ture. Constrain the rise in internal cell temperature by reducing the } C_{\text {rate }} \text {. On decreased } \\
\text { and stabilising the internal cell temperature, } C_{\text {rate }} \text { can be increased. }\end{array}$ \\
\hline 7 & Degraded & Low & Fast & $\begin{array}{l}\text { Start charging by deploying pulse charging with the discharge with amplitude of discharge } \\
\text { pulse higher than the average charge current. The rise in internal cell temperature should } \\
\text { be monitored. The charging should shift to a technique similar to CT5 on rise in temper- } \\
\text { ature. The internal cell temperature should be monitored and a reduction in } C_{\text {rate }} \text { should } \\
\text { be used to constrain the rise. }\end{array}$ \\
\hline 8 & Degraded & Normal & Fast & $\begin{array}{l}\text { The charging should be performed using a technique similar to CT5 and internal cell tem- } \\
\text { perature should be monitored. } C_{\text {rate }} \text { should be decreased to constrain the rise in internal } \\
\text { cell temperature after a certain safe value. }\end{array}$ \\
\hline 9 & Degraded & High & Fast & $\begin{array}{l}\text { The charging should start by a technique similar to CT5 and internal cell temperature } \\
\text { should be monitored. With an increase in the internal cell temperature above a certain } \\
\text { safe value, the } C_{\text {rate }} \text { should be reduced. The } C_{\text {rate }} \text { can be increased again once the cell } \\
\text { reaches the level of normal internal cell temperature. }\end{array}$ \\
\hline 10 & New & Low & Slow & $\begin{array}{l}\text { Start charging using a technique similar to pulse charging with the discharge with ampli- } \\
\text { tude of pulse discharge greater than the average charge current. The rise in temperature } \\
\text { should be monitored and with an increase, shift to a technique similar to CT5. The } C_{\text {rate }} \\
\text { should be decreased if the rise in internal cell temperature is observed around stability } \\
\text { limits. }\end{array}$ \\
\hline 11 & New & Normal & Slow & $\begin{array}{l}\text { A technique similar to CT5 should be used to charge and regular monitoring of rise in in- } \\
\text { ternal cell temperature should be done. The rise in internal cell temperature near stability } \\
\text { limits should be constrained by decreasing } C_{\text {rate }} \text {. }\end{array}$ \\
\hline 12 & New & High & Slow & $\begin{array}{l}\text { Start charging using a technique similar to CT5 and monitor the rise in internal cell tem- } \\
\text { perature. Reduce } C_{\text {rate }} \text { to control the rise in internal cell temperature. }\end{array}$ \\
\hline 13 & Half-life & Low & Slow & $\begin{array}{l}\text { The charging should start using a pulse charging with the discharge with amplitude of } \\
\text { pulse discharge greater than the average charge current. The rise in internal cell tempera- } \\
\text { ture should lead to shifting to CT5 or CT8. Reduce } C_{\text {rate }} \text { to control the rise in internal cell } \\
\text { temperature. }\end{array}$ \\
\hline 14 & Half-life & Normal & Slow & $\begin{array}{l}\text { The charging should be done using a technique similar to CT5. The internal cell tempera- } \\
\text { ture should be monitored and } C_{\text {rate }} \text { should be changed to constrain the rise in internal cell } \\
\text { temperature. }\end{array}$ \\
\hline 15 & Half-life & High & Slow & $\begin{array}{l}\text { Start charging using a technique similar to CT5 and monitor internal cell temperature. } \\
\text { Modify the } C_{\text {rate }} \text { to control the rise in internal cell temperature. }\end{array}$ \\
\hline 16 & Degraded & Low & Slow & $\begin{array}{l}\text { Start charging using pulse charging with the discharge with amplitude of discharge pulse } \\
\text { higher than the average charge current. Monitor the rise in internal cell temperature, and } \\
\text { shift charging to CT5 once temperature rises. The internal cell temperature should be } \\
\text { monitored to modify the } C_{\text {rate }} \text { on rise around safety limits. }\end{array}$ \\
\hline 17 & Degraded & Normal & Slow & $\begin{array}{l}\text { A A charging technique similar to CT5 should be performed with monitoring of inter- } \\
\text { nal cell temperature. The } C_{\text {rate }} \text { should be modified to constrain the rise in internal cell } \\
\text { temperature aß20 safety limits. }\end{array}$ \\
\hline 18 & Degraded & High & Slow & $\begin{array}{l}\text { Start charging similar to CT5 and monitor the rise in internal cell temperature. With an } \\
\text { increase in the internal cell temperature around safety limits, the } C_{\text {rate }} \text { should be reduced } \\
\text { and on reaching normal internal cell temperature, } C_{\text {rate }} \text { can be ageing increased. }\end{array}$ \\
\hline
\end{tabular}




\section{References}

1. G. E. Blomgren, Journal of The Electrochemical Society 164, A5019 (2016).

2. D. Kushnir, Current State and Future Prospects pp. 1-56 (2015).

3. N. Nitta, F. Wu, J. T. Lee, G. Yushin, Materials Today 18, 252 (2015).

4. A. Masias, J. Marcicki, W. A. Paxton, ACS Energy Letters 6, 621 (2021).

5. L. Ellingsen, C. Hung, STUDY, European Parliament, Directorate General for Internal Policies, Policy Department for Structural and Cohesion Policies, Transport and Tourism 10, 944056 (2018).

6. F. Conte, e \& i Elektrotechnik und Informationstechnik 123, 424 (2006).

7. P. Keil, et al., Journal of The Electrochemical Society 163, A1872 (2016).

8. J. Vetter, et al., Journal of power sources 147, 269 (2005).

9. M. R. Palacín, Chemical Society Reviews 47, 4924 (2018).

10. X. Han, et al., eTransportation 1, 100005 (2019).

11. V. Etacheri, R. Marom, R. Elazari, G. Salitra, D. Aurbach, Energy \& Environmental Science 4, 3243 (2011).

12. G. Sikha, B. N. Popov, R. E. White, Journal of the Electrochemical Society 151, A1104 (2004).

13. P. Arora, R. E. White, M. Doyle, Journal of the Electrochemical Society 145, 3647 (1998).

14. J. Liu, et al., Journal of Power Sources 445, 227263 (2020). 
15. P. Ramadass, B. Haran, P. M. Gomadam, R. White, B. N. Popov, Journal of the Electrochemical Society 151, A196 (2004).

16. D. Zhang, et al., Journal of Power Sources 91, 122 (2000).

17. P. Ramadass, B. Haran, R. White, B. N. Popov, Journal of power sources 112, 606 (2002).

18. P. Keil, A. Jossen, Journal of Energy Storage 6, 125 (2016).

19. G. Sikha, P. Ramadass, B. Haran, R. E. White, B. N. Popov, Journal of power sources 122, 67 (2003).

20. L. Patnaik, A. Praneeth, S. S. Williamson, IEEE Transactions on Industrial Electronics 66, 1059 (2018).

21. B. Balagopal, M.-Y. Chow, Batteries 6, 53 (2020).

22. M. H.-M. Tang, Side reactions in lithium-ion batteries, Ph.D. thesis, UC Berkeley (2012).

23. X. Zhang, A. M. Sastry, W. Shyy, Journal of The Electrochemical Society 155, A542 (2008).

24. S. Pelletier, O. Jabali, G. Laporte, M. Veneroni, Transportation Research Part B: Methodological 103, 158 (2017).

25. L. Lu, X. Han, J. Li, J. Hua, M. Ouyang, Journal of power sources 226, 272 (2013).

26. C. M. Snyder, The rate depedency of li-ion battery degradation mechanisms., Tech. rep., Sandia National Lab.(SNL-NM), Albuquerque, NM (United States) (2016).

27. R. Xiong, Y. Pan, W. Shen, H. Li, F. Sun, Renewable and Sustainable Energy Reviews 131, 110048 (2020). 
28. M. F. Hasan, C.-F. Chen, C. E. Shaffer, P. P. Mukherjee, Journal of the Electrochemical Society 162, A1382 (2015).

29. D. H. Doughty, C. C. Crafts, Freedomcar: electrical energy storage system abuse test manual for electric and hybrid electric vehicle applications., Tech. rep., Sandia National Laboratories (2006).

30. X. Zhou, J. Huang, Z. Pan, M. Ouyang, Journal of Power Sources 426, 216 (2019).

31. A. Andersson, K. Edström, Journal of the Electrochemical Society 148, A1100 (2001).

32. M. Richard, J. Dahn, Journal of The Electrochemical Society 146, 2068 (1999).

33. H. Maleki, G. Deng, A. Anani, J. Howard, Journal of The electrochemical society 146, 3224 (1999).

34. D. MacNeil, D. Larcher, J. Dahn, Journal of the electrochemical society 146, 3596 (1999).

35. P. Arora, M. Doyle, R. E. White, Journal of The Electrochemical Society 146, 3543 (1999).

36. C.-K. Huang, J. Sakamoto, J. Wolfenstine, S. Surampudi, Journal of the Electrochemical Society 147, 2893 (2000).

37. L.-R. Chen, IEEE Transactions on Industrial Electronics 54, 398 (2007).

38. B. Sah, P. Kumar, R. Rayudu, S. K. Bose, K. P. Inala, IEEE Transactions on Industrial Informatics 15, 3923 (2018).

39. B. Sah, P. Kumar, S. K. Bose, IEEE Systems Journal (2020).

40. M. Muratori, Nature Energy 3, 193 (2018). 


\section{Acknowledgments}

This work is supported by the Electric Mobility lab of the Department of Electronics and Electrical Engineering, Indian Institute of Technology Guwahati. The author would like to extend their thanks to the developers of Python Battery Mathematical Modelling (PyBaMM), specifically Dr Valentin Sulzer, for his support while preparing models and codes for implementing charging techniques. Further, the authors thank the Office of Dean of Industrial Interactions and Special Initiatives, Indian Institute of Technology Guwahati for their support in the Indian patent application No.202131028110 A.

\section{Funding}

The work is funded by the Electric Mobility lab of the Department of Electronics and Electrical Engineering, Indian Institute of Technology Guwahati.

\section{Author Contributions}

Bikash Sah and Praveen Kumar conceived and designed the research. Bikash Sah acquired the data, implemented the physics-based model of Li-ion battery, integrated the models of battery degradation, designed types of charging techniques, performed the data analysis and visualisation. Both the authors contributed to discussing results and writing the manuscript.

\section{Competing Interests}

The authors declare that they have no competing interests.

\section{Data and materials availability}

The data and materials are available on request from the corresponding author at praveen_kumar@iitg.ac.in. 